\section{Quoi de neuf aux éditions EMH?}

Dans le $n^{\circ} 24$ de cette année [1], nous vous avons présenté, chers lecteurs, nos éditions EMH, vous faisant part, notamment, des nouveaux projets qui nous tenaient à cœur. Le dernier numéro du "Bulletin des médecins suisses" vous relate les premiers signes de l'évolution en cours [2].

Un "Swiss Medical Weekly" plus qu'en anglais? La suppression - à nouveau - de la formation continue dans le BMS? Cette maison d'édition n'a t-elle donc plus aucun concept? Rassurez-vous, au contraire. Dès sa création, on esquissa des objectifs $\dot{a}$ court et à plus long terme. En tant qu'éditeur du corps médical suisse, les EMH s'engagent principalement à développer pour vous, lecteurs et auteurs, des produits de très haute qualité. La réalisation d'objectifs audacieux ne saurait cependant porter atteinte au bénéfice qui, par le biais d'une contribution annuelle à la FMH, sert aussi indirectement à abaisser les cotisations de membres. Après deux premières années couronnées de succès, nous sommes devant une autre étape à franchir. La réorganisation implique quelques changements dans nos publications.

Pourquoi donc un tel remaniement en profondeur? Il s'agit en premier lieu de mettre au net la teneur des publications en soi. De deux revues, on passe $\dot{a}$ trois: le "Bulletin des médecins suisses" pourra se concentrer sur ses thèmes centraux; le nouveau "Swiss Medical Weekly", en anglais seulement, a pour but de servir de forum pour les scientifiques d'orientation clinique; le nouveau "Forum médical suisse" présente les articles de formation continue du SMW et du BMS sous une nouvelle forme, structurée et orientée sur la pratique. Une autre nouveauté particulièrement intéressante sera de permettre à tous les médecins de lire ces documents concernant la formation continue sur le site internet de la revue avant la publication imprimée, d'exprimer leurs souhaits, d'apporter leurs suggestions et commentaires dont on peut ensuite tenir compte dans la rédaction définitive des textes.
A partir de 2001, des concepts clairs vous permettront, à vous lecteurs comme à vous auteurs, de connaître plus facilement le contenu de ces publications.

D'autres projets et organes de publication des sociétés de discipline médicale entièrement indépendants couronnent le tout. Un premier exemple se trouve être la "Médecine cardiovasculaire".

Une autre nouveauté a été présentée la semaine dernière à Montreux: "PrimaryCare", un produit commun aux EMH, à la Société Suisse de Médecine Générale (SSMG) et au Collège de médecine de premier recours (CMPR). Tous les membres des sociétés fondatrices du CMPR (SSMG, SSMI et SSP) devraient bientôt en recevoir le premier numéro. A partir de janvier 2001, "PrimaryCare" paraîtra tous les quinze jours et portera sur tous les sujets de la médecine de premier recours.

Il va de soi que, hormis les versions sur papier, toutes ces publications se retrouveront sur l'internet, sans frais, sans restrictions, in extenso, avec toutes les illustrations, les fonctions de recherche et d'archives. Ce n'est pas vraiment nouveau. Sur les sites http://www.bullmed.ch et http://www.smw.ch, vous trouvez déjà tout cela ou presque. Les journaux imprimés et ceux sur l'internet se complètent fort bien. Personne n'aurait l'idée (du moins pas encore) de ne feuilleter une revue que sur l'écran. Mais si vous cherchez un article des mois après sa parution, il n'existe guère de moyen plus pratique que l'internet pour le trouver, à condition que le site soit bien conçu.

L'article sur notre maison d'édition, dans le dernier numéro du BMS [2], a pour intention de vous inciter à vous révéler en tant qu'auteur pour le SMW ou le FMS. En nous réjouissant de votre future participation, nous répondrons volontiers $\dot{a}$ vos questions.

Editores Medicorum Helveticorum Ludwig T. Heuss et Natalie Marty

1 Aubert C, Bienz R, Breitenstein U, Düblin M, Heuss LT, Marty N, Trutmann M. EMH - l'éditeur des médecins. Bull Med Suisse 2000;81(24):1289-90.

2 Straub PW, Schaffner A, Krapf R. Le nouveau Swiss Medical Weekly et le nouveau Forum Médical Suisse. Bull Med Suisse 2000;81(35):1931-7. 